\title{
Evolución clínico-funcional en pacientes tratados mediante artroplastía total de rodilla sin estabilizador posterior vs con estabilizador posterior en el Centenario Hospital Miguel Hidalgo
}

\author{
Del Toro Lomelí Francisco *, Gutiérrez Mendoza Israel **, \\ Barba-Gallardo Luis Fernando**, Martínez-Hernández Ángel ****
}

\begin{tabular}{|c|c|}
\hline $\begin{array}{l}\text { Resumen } \\
\text { Introducción: En grados avanzados de la artrosis de rodilla el } \\
\text { tratamiento adecuado es la artroplastia total de rodilla (ATR), } \\
\text { una discusión frecuente es acerca de utilizar estabilizador } \\
\text { posterior o no en la ATR, dicha elección se fundamenta en } \\
\text { indicaciones quirúrgicas o en elección del cirujano para reali- } \\
\text { zarla. El objetivo de este estudio es comparar clínica y funcio- } \\
\text { nalmente resultados de la ATR con estabilizador posterior vs } \\
\text { sin estabilizador posterior. Método: Diseño clínico controlado, } \\
\text { siego simple, se operaron I } 8 \text { pacientes, con diagnóstico de } \\
\text { gonartrosis grado IV según escala radiográfica de Kellgreen- } \\
\text { Lawrence, se formaron dos grupos (con y sin estabilizador) } \\
\text { aleatoriamente, los pacientes fueron valorados por escore de } \\
\text { la sociedad de rodilla americana mensualmente en el pos- } \\
\text { quirúrgico. Resultados: Los resultados coinciden con lo descrito } \\
\text { por otros autores en trabajos similares; sin diferencias estadís- } \\
\text { ticamente significativas en los aspectos clínicos y funcionales } \\
\text { entre los pacientes tratados con o sin estabilizador. Conclusión: } \\
\text { Los pacientes con gonartrosis que requieren artroplastia total } \\
\text { de rodilla logran resultados de muy buenos a excelentes, ya } \\
\text { sea con o sin estabilizador posterior. LUX MÉDICA AÑo 7, NúmERo } \\
22 \text {, SEPTIEMBRE-DICIEMBRE } 2012 \text { PP } 19 \text {-24 }\end{array}$ & $\begin{array}{l}\text { Abstract } \\
\text { Background: In advanced stages of osteoarthritis of knee pro- } \\
\text { per treatment is total knee arthrolasty (AKT), a common dis- } \\
\text { cussion is about using posterior stabilization AKT or not used, } \\
\text { that choice is based on indications for surgery or surgeon's } \\
\text { choice to do it.The objective of this study was to compare clini- } \\
\text { cal and functional results of posterior stabilizerAKT vs no rear } \\
\text { stabilizer. Method: Controlled trial design, simple blind, it ope- } \\
\text { rated I } 8 \text { patients diagnosed with knee osteoarthritis grade } \\
\text { IV according to radiographic scale Lawrence-Kellgreen, were } \\
\text { divided in two groups (with and without stabilizer) randomly, } \\
\text { patients were assessed by score of American Knee Society } \\
\text { monthly in the postoperative, statistical analysis was paired } \\
\text { test and ANOVA. Results: The results are consistent with those } \\
\text { described by other authors in similar but clinically when com- } \\
\text { paring both groups were no differences in the function and } \\
\text { evolution, showing improvements. Conclusion: Patients with } \\
\text { knee osteoarthritis requiring total knee arthroplasty achieve } \\
\text { very good to excellent results, either with or without rear sta- } \\
\text { bilizer. LUX MÉDICA AÑO 7, NúMERO 22, SEPTIEMBRE-DICIEMBRE } 2012 \\
\text { PP } 19 \text {-24 }\end{array}$ \\
\hline Palabr & $\begin{array}{l}\text { throlasty, posterior stabilization, osteoar- } \\
\text { ent }\end{array}$ \\
\hline
\end{tabular}

Residente de cuarto año de la especialidad de Traumatología y Ortopedia del Centenario Hospital Miguel Hidalgo, Aguascalientes, Ags. México

** Médico Traumatólogo y Ortopedista Coordinador de Investigación del ISSEA

*** Profesor Investigador del Centro de Ciencias de la Salud UAA.

**** Profesor Adscrito al Servicio de Traumatología y Ortopedia del Centenario Hospital Miguel Hidalgo, Aguascalientes, Ags. México

Fecha de recibido 30 de mayo 2012

Fecha de aceptación 30 de septiembre de 2012

Correspondencia: Dr Israel Gutiérrez Mendoza, médico traumatólogo, investigador del Instituto de Salud del Estado de Aguascalientes. Margil de Jesús \# 1501, fracciona miento Arboledas CP 20020 Aguascalientes, Ags. México Teléfono 01 (449) 9107900 correo electrónico drgutierrez1@yahoo.com.mx,

deltorolomeli@hotmail.com 


\section{Introducción}

En algún momento de la evolución de las enfermedades incapacitantes de la rodilla, como la osteoartritis, el único tratamiento disponible será la operación para la colocación de una prótesis de la rodilla. Durante la artroplastia total de rodilla (ATR), dependiendo de la técnica y del cirujano, varias estructuras de la rodilla pueden ser conservadas, reemplazadas por estructuras artificiales o simplemente desechadas. ${ }^{1-3}$

En términos generales, al realizar una ATR se realizan cortes a nivel femoral y tibial, además de retirar el ligamento cruzado anterior (LCA), dejando el ligamento cruzado posterior (LCP) ya que este último nos dará la estabilidad posterior de la rodilla, y el LCA será suplido por el diseño de los componentes tibial y femoral de la prótesis; sin embargo, en algunos casos, se puede sacrificar el LCP y colocar un estabilizador posterior. Existen diferentes técnicas y tipos de implantes para realizar la ATR y uno de los temas que causan mayor controversia es si se debe conservar o no el LCP. ${ }^{4} \mathrm{Si}$ el LCP es resecado, se debe colocar una prótesis que cuente con estabilizador posterior. La tabla 1 muestra los factores que influyen en la elección para el sacrificio o la conservación del LCP. ${ }^{5}$

\section{Tabla I}

Factores que influyen en la elección para el sacrificio o la conservación del LCP

\begin{tabular}{|l|}
\hline Pérdida tensil del LCP o adelgazamiento mayor al $50 \%$, \\
\hline Grado de artrosis \\
\hline Sección trans quirúrgica iatrogénica con imposibilidad de reparación \\
\hline Contractura en flexión mayor a 20 grados \\
\hline Deformidad en varo mayor a $15^{\circ}$ \\
\hline Deformidad en valgo mayor a $10^{\circ}$ \\
\hline Tipo de implante utilizado en la cirugía \\
\hline Preferencia personal del cirujano \\
\hline
\end{tabular}

En la práctica, la evaluación del éxito de la ATR se hace mediante la clasificación de la American Knee Asociation (AKA), que hace uso de dos aspectos que son: la función (la puntuación del paciente) y la clínica (la puntuación del cirujano). Basados en la clasificación AKA se puede clasificar el resultado de la cirugía en resultados: malos, regulares, buenos o excelentes. ${ }^{6}$

El objetivo de este estudio fue el comparar el resultado clínico-funcional de la ATR en pacientes operados con y sin la colocación de estabilizador posterior.

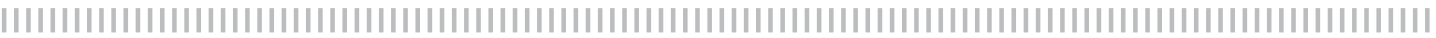

\section{Material y métodos}

Se realizó un ensayo clínico aleatorizado, en el periodo comprendido del 1 de enero 2010 al 15 de noviembre 2011, en el cual se incluyeron pacientes mayores de 55 años de ambos géneros con diagnóstico de gonartro- sis grado III y IV de la clasificación radiográfica de Kellgreen-Lawrence que requirieron tratamiento quirúrgico mediante ATR. Los pacientes fueron asignados de forma aleatoria a recibir la ATR sin estabilizador posterior (SEP) o con estabilizador posterior (CEP). 
La técnica quirúrgica en todos los pacientes fue con abordaje anterior y artrotomía medial, la prótesis utilizada en todos los casos fue la fabricada por Johnson \& Johnson correspondiente a la segunda generación; todos los pacientes llevaron un programa de ejercicios de rehabilitación (isométricos e isotónicos) de manera seriada cada cuatro horas y deambulación asistida con andadera posterior a la cirugía.

Se realizó evaluación clínico-funcional pre-quirúrgica y post-quirúrgica mensual

\section{| | | | | | | | | | | | | | | | | | | | | | | | | | | | | | | | | | | | | | | | | | | | | | | | | | | | | | | | | | | | | | | | | | | | | | | | | | | | | | | | | | | | | | | | | | | | | | | | | | | | | | | | | | | | | | | | | | | | | |}

\section{Resultados}

En el periodo comprendido de 1 de enero 2010 al 15 de noviembre del 2011 fueron aleatorizados 18 pacientes en el estudio, todos cumplían los criterios de inclusión y fueron operados de ATR. No se presentó ninguna eliminación durante el estudio. La mayoría fueron mujeres con $83.3 \%$ (15 pacientes). La edad media fue de $66.9 \pm$ 9 años con una mínima de 55 años y un máximo de 83 años.

La rodilla mayormente afectada fue la de-

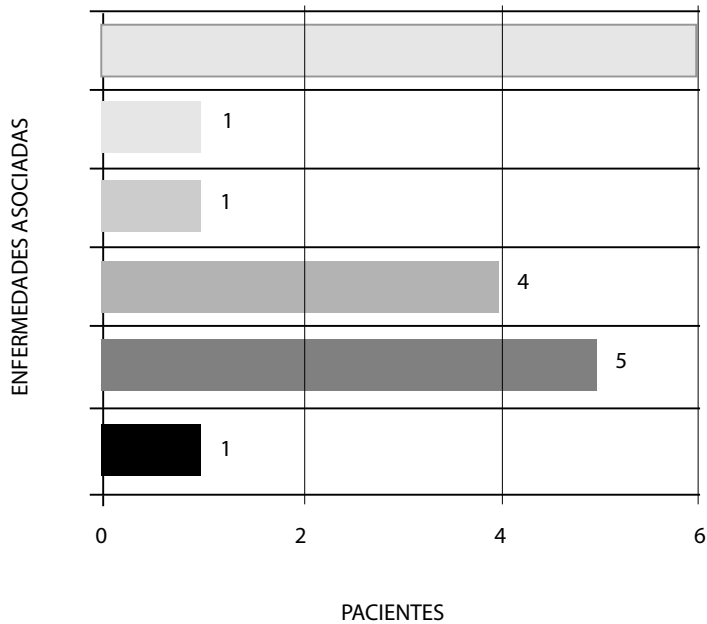

El tiempo quirúrgico promedio en pacientes SEP fue de $122 \pm 26$ minutos y en aquellos sometidos a CEP fue de $113 \mathrm{mi}$ nutos \pm 21 minutos, siendo significativamente menor el tiempo quirúrgico en los pacientes CEP. hasta los seis meses, empleando la escala de valoración de la AKA. Todos los pacientes asistieron a centro de rehabilitación profesional asistida por médico especialista 15 días después de evento quirúrgico, tres veces por semana y durante los seis meses en que se evaluaron. Se realizaron comparaciones estadísticas entre los grupos por medio de la prueba $T$ para muestras independientes y ANOVA de una vía. Los análisis se llevaron a cabo mediante el paquete estadístico PASW Statistics ${ }^{18}$.

recha en 10 pacientes (55.6\%), a comparación con la izquierda con un promedio de 8 pacientes (44.4\%). La ocupación más frecuente fue ama de casa, 14 pacientes $(77.8 \%), 3$ pacientes $(16.7 \%)$ se dedican a la agricultura y 1 paciente $(5.6 \%)$ fue profesionista. El $33.3 \%$ (6 pacientes) no se presentaron enfermedades asociadas, mientras que el $66.7 \%$ tuvieron alguna comorbilidad, la figura 1 muestra el histograma de frecuencias de estas comorbilidades. El $100 \%$ de los pacientes eran diestros.

\begin{tabular}{|c|c|}
\hline \multicolumn{2}{|l|}{ NINGUNO 33.3\% } \\
\hline AR 5.6\% & Figura 1. Comorbilidades \\
\hline IVP 5.6\% & $\begin{array}{l}\text { asociadas en pacientes } \\
\text { llevados a artroplastía }\end{array}$ \\
\hline HAS Y DM $22.2 \%$ & total de rodilla \\
\hline \multicolumn{2}{|l|}{ HAS $28.8 \%$} \\
\hline \multicolumn{2}{|l|}{ DM 5.6\% } \\
\hline $\begin{array}{l}\text { Artritis reumatoi } \\
\text { Insuficiencia ven } \\
=\text { Hipertensión a } \\
\text { Diabetes mellit }\end{array}$ & $\begin{array}{l}\text { rofunda } \\
\text { al sistémica } \\
\text { bo } 2\end{array}$ \\
\hline
\end{tabular}

No hubo diferencias significativas al comparar el sangrado trans quirúrgico, el sangrado postquirúrgico, ni el sangrado total entre ambos grupos (Tabla 1). 


\section{Tabla I}

Sangrado comparativo en mililitros.

\begin{tabular}{|lcc|c|}
\hline & Sin estabilizador & Con estabilizador & P \\
\hline Trans quirúrgico & $261 \pm 155 \mathrm{ml}$ & $214 \pm 126 \mathrm{ml}$ & 0.436 \\
\hline Posquirúrgico & $98 \pm 32 \mathrm{ml}$ & $74 \pm 20 \mathrm{ml}$ & 0.073 \\
\hline TOTAL & $360 \pm 171 \mathrm{ml}$ & $288 \pm 121 \mathrm{ml}$ & 0.325 \\
\hline
\end{tabular}

Todos los valores promedio \pm desviación estándar.

La tabla 2 muestra las puntuaciones obtenidas en la escala de la AKA a lo largo de las evaluaciones mensuales, el las cuales a pesar de existir una tendencia a mejores puntuaciones en el grupo CEP, éstas no alcanzaron significado estadístico.

\section{Tabla 2}

Puntaje en la escala de la AKA a lo largo del seguimiento.

\begin{tabular}{|lcccc|}
\hline \multicolumn{4}{c}{ Clínico } & Funcional \\
\hline Preqx & Sin estabilizador & Con estabilizador & Sin estabilizador & Con estabilizador \\
\hline 1er mes & $41 \pm 20$ & $29 \pm 12$ & $34 \pm 11$ & $46 \pm 22$ \\
\hline 2do mes & $49 \pm 9$ & $66 \pm 15$ & $13 \pm 9$ & $29 \pm 25$ \\
\hline 3er mes & $66 \pm 14$ & $81 \pm 14$ & $45 \pm 7$ & $58 \pm 22$ \\
\hline 4to mes & $73 \pm 15$ & $83 \pm 15$ & $55 \pm 13$ & $72 \pm 23$ \\
\hline 5to mes & $80 \pm 11$ & $89 \pm 14$ & $68 \pm 17$ & $83 \pm 21$ \\
\hline 6to mes & $83 \pm 9$ & $89 \pm 15$ & $78 \pm 16$ & $88 \pm 15$ \\
\hline
\end{tabular}

Todos los valores promedio \pm desviación estándar. Resultado de la prueba T para muestras independientes no significativa en todos los casos.

En 3 pacientes $(16.8 \%)$ se presentaron complicaciones, una paciente tuvo una caída postquirúrgica en la cual se evidenció una regresión en los arcos de movilidad sin afectar a la función de la rodilla, en otra paciente hubo contractura en flexión, y en otra paciente hubo una celulitis superficial, la cual remitió después de 15 días de antibiótico terapia vía oral.

\section{| | | | | | | | | | | | | | | | | | | | | | | | | | | | | | | | | | | | | | | | | | | | | | | | | | | | | | | | | | | | | | | | | | | | | | | | | | | | | | | | | | | | | | | | | | | | | | | | | | ||}

\section{Discusión}

El retiro o conservación del LCP constituye una fuente actual de controversia médica pues en distintas partes del mundo el asunto es manejado de forma diferente, por ejemplo, la escuela italiana justifica el sacrificio del LCP basándose en estudios de patología donde se observa que el liga- mento pierde su fuerza tensil cubriéndose de tejido fibroso y al retirarlo se tiene una mejor estabilización posterior con la prótesis, además de mejorar los arcos de movilidad de los pacientes, ya que aumenta un $6,4 \mathrm{~mm}$ de la amplitud de flexión de la rodilla. ${ }^{7}$ Sin embargo en otras escuelas, como la americana o francesa la tendencia 
es a conservar el LCP celosamente, argumentando que el LCP logra mejoría clínica al subir escaleras debido al roll back que tiene la biomecánica de la rodilla y al colocar un estabilizador posterior sacrificando el LCP los pacientes tienen la sensación de inestabilidad al realizar esta actividad. ${ }^{8}$

Los pacientes evaluados en este estudio, coinciden con la distribución de sexo y edad de presentación en México según la Organización Mundial de la Salud (OMS), constituyéndose como una de las principales enfermedades degenerativas no metabólicas de adultos mayores a nivel mundial.

El tiempo quirúrgico obtenido, es una variable muy importante para la toma de decisiones trans-quirúrgicas, ya que algunos cirujanos prefieren el uso de isquemia neumática programada, por lo que el tiempo quirúrgico es favorable para el control trans y postquirúrgico disminuyendo el dolor, infección o enfermedades trombóticas locales y sistémicas, en este caso nuestros resultados fueron menor tiempo quirúrgico en los pacientes con estabilizador posterior que los sin estabilizador posterior, esto se debe a que la técnica quirúrgica es más sencilla al colocar el estabilizador ya que al realizar el corte tibial se hace sin tener que cuidar el LCP que ya se resecó previamente, además al retirarlo la rodilla se abre mas, lo que se conoce como gap por lo que resulta más fácil cementar los componentes protésicos, es importante señalar que en este estudio, a ningún paciente se le colocó isquemia durante el evento quirúrgico, nosotros explicamos nuestros resultados por la mayor sencillez de colocar el estabilizador posterior sacrificando el LCP.

La principal variable estudiada y objetivo principal de este trabajo fue la comparación clínica y funcional de los pacientes sometidos a ATR CEP y SEP, a este respecto pudimos observar que, por ejemplo, en la valoración prequirúrgica el grupo SEP logró mejores resultados que los del grupo CEP, este dato es importante ya que des- de la valoración prequirúrgica los pacientes del grupo SEP lograron un mayor puntaje según la escala de valoración de la AKA, y a pesar de esto, los resultados clínicos finales fueron más favorables para los pacientes CEP.

En conclusión, desde la valoración prequirúrgica el grupo SEP tuvieron mejores resultados clínicos, pero, desde el primer mes postquirúrgicos estos resultados fueron a la inversa logrando mejores resultados desde el primer mes hasta el sexto mes los pacientes CEP, por lo que lograron mayores arcos de movilidad desde el postquirúrgico con estabilizador posterior a pesar que este grupo de pacientes en el prequirúrgico tenían peores arcos de movilidad que los del grupo que no se les coloco estabilizador posterior. Las inferencias anteriores denotan tendencias, las cuales sin embargo no alcanzaron significado estadístico.

Al compararlo con la literatura actual la clínica posterior a una ATR en pacientes CEP y SEP es similar a la de la serie reportada por Cloutier et al, ${ }^{9}$ quien describe que existen diferencias en cuanto a la clínica pero no a la función, sus pacientes también lograron mayores arcos de movilidad lo que el explica por el mayor espacio entre el componente femoral respecto al tibial o gap. Otro estudio por Tanzer et al, ${ }^{10}$ demuestra similitudes de función, ya que los pacientes mejoraron clínicamente, se enfocaron principalmente en la biomecánica de la prótesis estabilizada, haciendo saber que el gap y el traking de la rodilla en flexión no afecta la función como se cree, y las prótesis estabilizadas tienen como base un mayor gap por ende un mayor arco de movilidad, sin disminuir o aumentar la función de la rodilla.

Nuestros resultados en cuanto a la función de ambos grupos fue a la inversa que la valoración clínica, los pacientes SEP tuvieron menor puntaje que los CEP desde la valoración inicial pre-quirúrgica, y los resul- 
tados funcionales obtenidos subsecuentemente fueron a la par, solamente tuvimos diferencias significativas del grupo CEP en el segundo mes logrando mayor puntaje, pero ya nuestros resultados finales fueron similares logrando ambos grupos un puntaje de muy bueno a excelente según la escala KSS.

Misra et al ${ }^{11}$ opinan diferente en su publicación acerca de la función, concluyendo que mejora significativamente la función en pacientes con prótesis estabilizada, además de mejorar también los arcos de movilidad, la estabilidad, la estrechez de la rodilla y reduce el estrés de la interface hueso-prótesis. Esto lo explican por la mejoría en cuanto a la estrechez de la rodilla, ya que consideran que la rodilla no queda constreñida mejorando así los arcos de movilidad y la función. Nosotros podemos coincidir en la mejoría de los arcos de movilidad sin embargo no encontramos diferencias en cuanto a la función, la estabilidad y la estrechez de la rodilla.

Basados en nuestros resultados, no podemos hacer una recomendación entre la conservación o el sacrificio del LCP colocando un componente estabilizador posterior ya que ambos grupos estudiados tuvieron mejoría significativa según la escala AKA independientemente de que el tratamiento hubiera sido CEP o SEP.

\section{Conclusiones}

Los pacientes con gonartrosis que requieren ATR logran resultados muy buenos a excelentes, ya sea CEP o SEP según la escala clínico-funcional de la AKA; sin embargo, los pacientes tratados CEP lograron mejores resultados clínicos que aquellos con SEP, aun cuando no existieron diferencias estadísticamente significativas respecto a la función al comparar ambos grupos.

\section{Bibliografía}

1. Francisco Javier Pasos El tratamiento del dolor en la gonartrosis. Federación y Colegio Nacional de Ortopedia. Vol. 23. México. P17-22.

2. Gonartrosis, Diagnóstico, tratamiento artroscópico y seguimiento. 14 edición. Madrid. Mc Graw-Hill, Interamericana de España, S.A.U. 1998; II: 2191.

3. I. Morgado. "Guía de Manejo Clínico de la Artrosis de Cadera y Rodilla". Rev. Soc. Esp. Dolor. 2005; 12(289):289-302.

4. Stephen A. Mikulak, MD. Posterior Stabilized Prosthesis. The Journal of Bone and Joint Surgery, 2001; 83: 398

5. Carlos J. Lavernia, Dr. José Carlos Alcerro. Artroplastia Total de Rodilla. Actualidades de Postgrado para el Médico. Orthopaedic Institute at Mercy Hospital. Miami, F1. EUA. Septiembre 2008, 13(7):

6. Campbell, Trastornos no traumáticos de origen diversos. Campbell Cirugía Ortopédica. Cap. 25. Ed Mosby. pp 223-242, Tomo III

7. Liow RY, Walker K, Wajid MA, Bedi G, Lennox CM. The reliability of the American Knee Society Score.
Acta Orthop Scand. 2000;71(6):603-8.

8. Chaudhary R, Beaupré LA, Johnston DW. Knee Range of Motion during the First Two Years after Use of Posterior Cruciate-Stabilizing or Posterior Cruciate Retaining Total Knee Prostheses A Randomized Clinical Trial. J Bone Joint Surg Am. 2008;90:25792586.

9. Emodi GJ, Callaghan JJ, Pedersen DR, Brown TD. Posterior cruciate ligament function following total knee arthroplasty: the effect of joint line elevation. lowa Orthop J. 1999;19:82-92.

10. J.M. Cloutier, Conservación de los Ligamentos Cruzados en las Artroplastias Totales de rodilla, Revista Española de Cirugía Osteoarticular, (397-402) 1990

11. Michel Tanzer, Posterior-Stabilized Versus Cruciate-Retaining Total Knee Arthroplasty, Balancing the Gap, The Journal of Arthroplasty Vol. 17 No. 7 October 2002

12. Misra AN, Hussain MR, Fiddian NJ, Newton G.Oscar J. Nordelo Martínez.The role of the posterior cruciate ligament in total knee replacement. J Bone Joint Surg Br. 2003 Apr;85(3):389-92. 